\title{
Experimental Multilevel NLOS Characterization for UWB Network Localization
}

\author{
Matteo Guerra and Andrea Conti \\ ENDIF, University of Ferrara \\ Via Saragat 1, 44100 Ferrara, Italy (e-mail: a. conti@ieee.org).
}

\begin{abstract}
Network localization is a new paradigm gaining interest in social, commercial, and military sectors. Ultrawide bandwidth (UWB) technology is promising for high-accuracy indoor localization due to the property of UWB signals. However, in non-line-of-sight (NLOS) conditions measurements are significantly biased, thus, high accuracy localization is challenging. We present a method for multilevel NLOS characterization, based on waveform measurements and their features, and we analyze its impact on localization error for both cooperative and noncooperative localization algorithms. The results enable a system designer to understand the benefits of cooperation and NLOS characterization on the localization performance.
\end{abstract}

Index Terms-Network localization, NLOS detection, ultrawide bandwidth (UWB), experimentation, ranging.

\section{INTRODUCTION}

The global positioning system (GPS) is currently the widest used technology enabling location-awareness. It provides localization accuracy on the order of meters in open outdoor areas, but fails in harsh operating environments such as inside buildings and urban canyons. In these cluttered environments multipath, line-of-sight (LOS) blockage, and excess propagation delay through materials present significant challenges to positioning. In these challenging environments, ultrawide bandwidth (UWB) transmission technology is a promising alternative for localization enabling accurate (mostly submetric) ranging and location estimation due to its ability to resolve multipath and penetrate obstacles [1]-[5]. Localization occurs in two main steps: measurements collection among nodes and processing through localization algorithms to determine the positions of agent nodes.

In time based ranging the distance between a pair of nodes is obtained using measurements of the signal propagation delay, or time-of-flight. This can be made using one-way time-ofarrival (TOA), two-way TOA, or time difference-of-arrival (TDOA) ranging techniques. A number of implementation challenges are present for UWB TOA estimation systems, such as noise, multipath effects, interference, clock drifts, and environmental effects as non-line-of-sight (NLOS) propagation [6]. NLOS propagation introduces positive bias to TOA, due to an additional delay of the signals through materials, which can seriously affect the localization performance [2], [7]. Harnessing environmental information, such as the identification of the NLOS conditions, in the presence of direct path (DP) excess delay and blockage, can improve localization performance by discarding or refining unreliable range measurements [8]. Several NLOS identification and mitigation methods for UWB positioning systems have been presented in literature [9], [10]. Non-parametric solutions for NLOS identification can be found in [11], [12]. Parametric solutions are mainly based on likelihood ratio test (LRT) with binary LOS/NLOS hypotheses and require the extraction of features from the received waveform [9], [13]. An overview of NLOS identification techniques can be found in [10]. The possibility of performing measurements between any pair of nodes enables the use of cooperation, where each agent uses range information not only from the anchors but also from other agents to extend coverage and improve localization accuracy [14]. The links between different agent nodes may be affected by significant ranging errors, thus it is of interest to jointly utilize environmental information and cooperation for network localization [15].

In this paper, we introduce a multilevel NLOS characterization method based on experimental data. The key features are extracted directly from the measured waveform and the maximum-likelihood (ML) estimator is considered which extends the classical binary LRT. The method proposed enables the statistical characterization of the number of walls present between each pair of nodes. We evaluate the impact of this technique on localization performance for cooperative and non-cooperative algorithms. As a case study, we consider the localization in realistic indoor environments where $N_{\mathrm{b}}$ anchors are deployed in known positions to determine the positions of $N_{\mathrm{a}}$ agents.

The remainder of this paper is organized as follows. In Section II, we briefly describe the measurement campaign and the ranging error model. In Section III, we propose a novel multilevel NLOS identification method with the experimental characterization of the waveform features of interest. In Section IV, we provide experimental results on localization accuracy, and our conclusion are given in Section V.

\section{Measurement Campaign and Ranging Models}

A measurement campaign has been performed at the WiLAB University of Bologna, Italy, to characterize UWB ranging behavior in a house with concrete walls. Ranging and waveform measurements have been performed with UWB commercial devices for a network of $N_{\mathrm{a}}=20$ agents (with identifiers 1-20) and $N_{\mathrm{b}}=5$ anchors (with identifiers B1-5), as in Fig. 1. A variety of situations LOS and NLOS with one or more walls is present in the considered environment.

Results from this measurement campaign suggest that we can model the range measurement $r_{i, j}$ between the pair of 


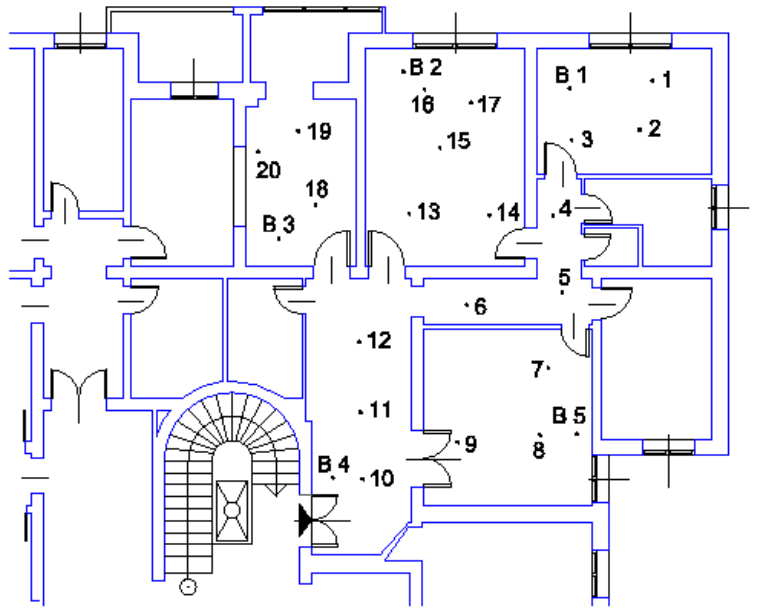

Fig. 1. Experimentation environment at Wilab, University of Bologna.

nodes in position $i$ and $j$ as

$$
r_{i, j}=d_{i, j}+b_{i, j}+\epsilon_{i, j}
$$

where $b_{i, j}$ is the bias introduced by the obstacles between the two nodes, and $d_{i, j}$ is the true distance. The error term $\epsilon_{i, j}$ can be modeled as a Gaussian random variable (RV), independent of $b_{i, j}$, with zero mean and variance $\sigma_{i, j}^{2}$ [8], [16]. The parameter $\sigma_{i, j}$ for the scenario considered is obtained from experimental measurements once the number of walls between the two nodes' positions is known [8] . In the absence of a priori information on the environment topology, all $\sigma_{i, j}$ are considered equal to the average over all possible pairs of nodes. The bias $b_{i, j}$ can be treated either as a RV, in case a statistical characterization is available [17], or as a deterministic quantity if it is somoehow known. In particular, we consider here a deterministic model named wall extra delay (WED). This is motivated by measurements that show a bias which depends primarly on the walls obstructing the DP signal and modeled as

$$
b_{i, j}=c \sum_{k=1}^{N_{\mathrm{w}}(i, j)} W_{k}^{(i, j)} \Delta_{k}
$$

where $c$ is the speed of light, the summation is over the $N_{\mathrm{w}}(i, j)$ different extra delay values between node $i$ and node $j$, and $W_{k}^{(i, j)}$ is the number of walls that result in the same extra delay value $\Delta_{k}$. The total number of walls separating the two nodes is $W^{(i, j)}=\sum_{k=1}^{N_{\mathrm{w}}(i, j)} W_{k}^{(i, j)}$. When every wall in the scenario has the same thickness and composition (i.e., $\Delta_{k}=\Delta$ for each $k$ ), (2) is simplified to $b_{i, j}=c W^{(i, j)} \Delta$. The wall extra delay (WED) model can be used for refining ranging and improving localization performance [8], [15].

\section{Multilevel NLOS Characterization}

The bias of the ranging estimates error degrades the localization performance. It is important to harness information on the environment topology, if available, to refine ranging estimates [8], [15]. In the considered scenario, we distinguish between five obstruction situations:
- LOS: absence of walls between the two nodes;

- $\operatorname{NLOS}_{i}$ : presence of $i$ walls between the two nodes (e.g., $i=1,2,3,4$ in the scenario considered).

We first give a brief description of the parametric approaches, and waveform features used to distinguish between possible conditions.

\section{A. NLOS Identification}

Most of the LOS/NLOS identification approaches, proposed in literature, are based on classical binary LRT [18]. To discriminate various environmental situations, we generalize classical method by means of ML estimation. For a received waveform we extract $N$ features $\gamma=\left\{\gamma_{1}, \gamma_{2}, \ldots, \gamma_{N}\right\}$ and apply the following decision criterion to estimate the number of obstacles encountered by the transmitted signal.

$$
\widehat{C}=\underset{x \in \mathcal{C}}{\arg \max } p(\gamma \mid x) \mathbb{P}\{x\}
$$

where $\mathcal{C}$ is the set of possible conditions, that is $\mathcal{C}=$ $\left\{\mathrm{LOS}, \mathrm{NLOS}_{1}, \mathrm{NLOS}_{2}, \mathrm{NLOS}_{3}, \mathrm{NLOS}_{4}\right\}, p(\gamma \mid x)$ is the joint PDF of features conditioned by $x$ and $\mathbb{P}\{x\}$ is the a priori probability of event $x$. Since this joint PDF is hard to obtain, in [18] a suboptimal solution was proposed to consider the features as independent, e.g. with three features the (3) becomes

$$
\widehat{C}=\underset{x \in \mathcal{C}}{\arg \max } p\left(\gamma_{1} \mid x\right) p\left(\gamma_{2} \mid x\right) p\left(\gamma_{3} \mid x\right) \mathbb{P}\{x\}
$$

In the absence of a priori information, that is $\mathbb{P}\{x\}$ is unknown, we can remove $\mathbb{P}\{x\}$ from the (4). The purpose of the next section is to evaluate, through experimental measurements, the term $p(\gamma \mid x)$ for all $x \in \mathcal{C}$ and features $\gamma$.

\section{B. Statistical Characterization of Waveform Features}

A key aspect for the design of the identification approach is the choice of the appropriate set $\gamma$ of signal features. A variety of features, extracted from a waveform $v(t)$ in an observation time interval $T$, have been proposed in the literature [11], [18]. We consider the following features:

1) Root mean square delay spread (RMS-DS), hereafter $\gamma_{1}$

$$
\tau_{\mathrm{rms}}=\left[\frac{\int_{T}\left(t-\tau_{\mathrm{m}}\right)^{2}|v(t)|^{2} d t}{\int_{T}|v(t)|^{2} d t}\right]^{1 / 2}
$$

where $\tau_{\mathrm{m}}$ is the mean excess delay defined as

$$
\tau_{\mathrm{m}}=\frac{\int_{T} t|v(t)|^{2} d t}{\int_{T}|v(t)|^{2} d t}
$$

2) Kurtosis (KUR), hereafter $\gamma_{2}$

$$
\mathcal{K}=\frac{1}{\sigma_{|v|}^{4} T} \int_{T}\left(|v(t)|-\mu_{|v|}\right)^{4} d t
$$

where $\mu_{|v|}$ and $\sigma_{|v|}^{2}$ are the mean and variance of $v(t)$.

3) Maximum amplitude (MA), hereafter $\gamma_{3}$

$$
v_{\max }=\max _{t \in T}|v(t)| .
$$

From the measurements we observe that (i) LOS signals are tipically less attenuated than NLOS signals; (ii) the RMS 


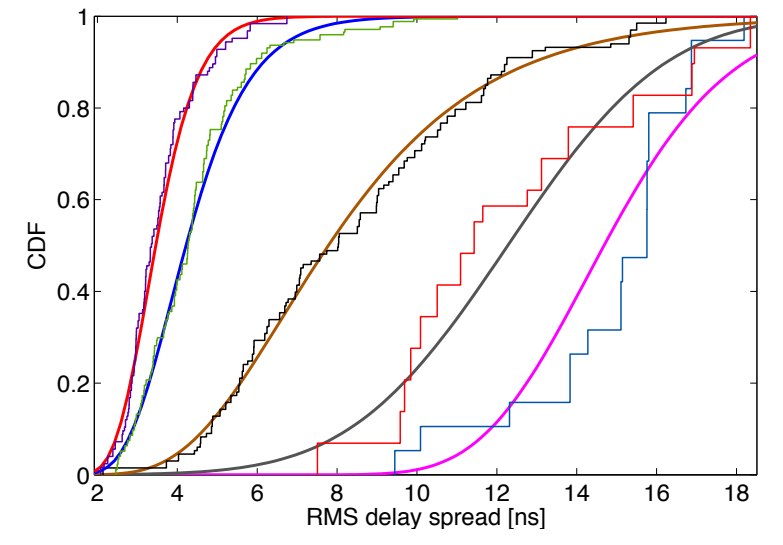

Fig. 2. CDF of the RMS delay spread for all possible condition of LOS and NLOS. Comparison with the Log-Normal statistics.

delay spread, that measures the temporal dispersion of the waveform, is larger in NLOS conditions; (iii) LOS waveforms generally show a higher value for the Kurtosis with respect to NLOS ones, as highlighted also in [11]. Experimental results show also that the probability density function (PDF) of these features for all environments conditions can be modeled as a log-normal distribution, with parameters that depend on the specific condition and feature considered. Fig. 2, shows the cumulative distribution functions (CDF) for RMS delay spread $\gamma 1$ measured in the LOS, $\mathrm{NLOS}_{1}, \mathrm{NLOS}_{2}, \mathrm{NLOS}_{3}$ and $\mathrm{NLOS}_{4}$. These CDFs are then compared to the log-normal $\mathrm{CDF}$ and in all cases show a clear agreement between the measured data and the log-normal model with mean and variance extracted from measurements.

Based on these considerations, a way to improve the localization performance is to follow these three localization steps: (i) obstacle detection through statistical identification of obstacles based on (4) and a combination of waveform features considered; (ii) range refinement through bias removal based on estimated number of walls in (i) and WED model in (2); (iii) position estimation with a localization algorithm based on refined (unbiased) ranging values.

\section{EXPERIMENTAL RESUlTS ON LOCALIZATION}

We now verify the impact of multilevel NLOS identification technique on cooperative and noncooperative localization based on measured waveform and ranging in the environment of Fig. 1. The measurements are elaborated by a localization algorithm, based on Bayesian estimation, which has been proposed in [17]. This algorithm, called sum-product algorithm over wireless network (SPAWN), has been derived by mapping a graphical model for statistical inference onto the network topology, which results in a network factor graph, and by developing a suitable network message passing scheduling. SPAWN is a Bayesian distributed, cooperative localization algorithm that can be used for different scenarios such as cooperative and non-cooperative, dynamic and static networks.

\section{A. Performance Metrics}

A local property, namely the localization root-mean-square error (RMSE), is given by the Euclidean distance between the
TABLE I

NON-COOPERATIVE LOCALIZATION RMSE $e(\mathbf{x})$ [CM] FOR VARIOUS WAVEFORM FEATURES (A)-(E).

\begin{tabular}{|l|l|l|l|l|l|}
\hline ID & $(\mathbf{A})$ & $(\mathbf{B})$ & $(\mathbf{C})$ & $(\mathbf{D})$ & $(\mathbf{E})$ \\
\hline \hline Mean & 46.65 & 24.23 & 37.02 & 20.04 & 21.53 \\
StD & 20.47 & 14.34 & 22.20 & 13.53 & 12.23 \\
Span & 62.46 & 44.56 & 75.22 & 49.71 & 41.56 \\
\hline
\end{tabular}

(A) No WED identification

(B) RMS delay spread (RMS-DS)

(C) Kurtosis (KUR)

(D) Maximum Amplitude (MA)

(E) MA-RMS-KUR jointly

estimated position $\hat{\mathrm{x}}$ and the true position $\mathrm{x}$ as

$$
e(\mathbf{x})=\|\hat{\mathbf{x}}-\mathbf{x}\|
$$

given a certain scenario in terms of the number of anchors $N_{\mathrm{b}}$ and their deployment, the number of agents $N_{\mathrm{a}}$, and the propagation conditions. We also evaluate the localization performance, in terms of outage probability (a figure of merit derived from that used in communication systems, see e.g. [19]). For a given target localization RMSE allowable error $e_{\text {th }}$, the agent in position $\mathbf{x}$ is in outage when its localization RMSE $e(\mathbf{x})$ exceeds $e_{\mathrm{th}}$, that is

$$
P_{\mathrm{o}}=\mathbb{P}\left\{e(\mathbf{x})>e_{\mathrm{th}}\right\}=\mathbb{E}\left\{\mathbb{I}\left\{e(\mathbf{x})>e_{\mathrm{th}}\right\}\right\}
$$

where $\mathbb{I}\{\cdot\}$ is the indicator function, which, is zero when the proposition is false and one otherwise, $\mathbb{E}\{\cdot\}$ represents the statistical expectation made over the ensemble of all possible spatial positions and time instants. This global performance index is called localization error outage (LEO).

\section{B. Performance Results}

We first evaluate the localization RMSE for the noncooperative case with the multilevel NLOS identification technique proposed in Section III-A based on the three features RMS-DS, KUR, MA defined in Section III-B and their joint evaluation. Results are reported in Tab I. The non-cooperative case without NLOS identification and ranging refinement is also reported (A). Note that the usage of ranging refinement improve significantly the performance. In particular, the feature MA shows the best average behavior of the RMSE, on the other hand the use of MA, RMS-DS and KUR jointly can reduce the localization RMSE standard deviation and span on the whole considered scenario. We then focus on the joint features for NLOS identification and we evaluate the impact of both cooperation and ranging refinement. In Tab II the localization RMSE is reported for various combinations of absence or presence of cooperation and ranging refinement. As cooperative setting we consider the case of agents 6 and 15 acting as cooperative nodes for all the other agents. For both cooperative and non-cooperative settings, we compare the performance with that of a system that ideally knows the WED present between each pair of nodes (referred to as "genius aided"). It can be noted that cooperation alone improve the performance in the average (compare columns $\mathrm{A}$ and D) as well as ranging refinement alone does (compare columns A, B, and C). The multilevel NLOS identification 
TABLE II

LOCALIZATION RMSE $e(\mathbf{x})$ [CM] FOR EACH NODE POSITION (ID) IN VARIOUS SITUATIONS $(\mathrm{A})-(\mathrm{F})$.

\begin{tabular}{|l|l|l|l|l|l|l|}
\hline ID & $(\mathbf{A})$ & $(\mathbf{B})$ & $(\mathbf{C})$ & $(\mathbf{D})$ & $(\mathbf{E})$ & $(\mathbf{F})$ \\
\hline \hline Mean & 46.65 & 21.53 & 19.87 & 36.88 & 20.17 & 17.60 \\
StD & 20.47 & 12.23 & 10.9 & 19.87 & 11.49 & 10.00 \\
Span & 62.46 & 41.56 & 44.68 & 73.90 & 35.19 & 38.30 \\
\hline
\end{tabular}

(A) Non-cooperative SPAWN without WED identification

(B) Non-cooperative SPAWN with WED identification

(C) Non-cooperative SPAWN with WED genius aided

(D) Cooperative SPAWN without WED identification

(E) Cooperative SPAWN with WED identification

(F) Cooperative SPAWN with WED genius aided

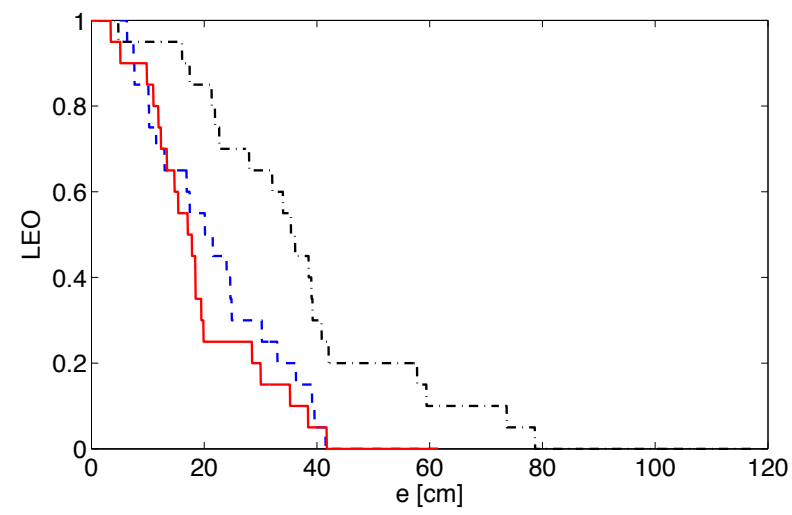

Fig. 3. LEO versus $e_{\mathrm{th}}[\mathrm{cm}]$ for cooperative SPAWN $N_{\mathrm{b}}=5$, cooperating nodes 6 and 15, with WED genius aided (red-continuous), with WED identification (blue-dashed), and without ranging refinement (black-dash-dot).

provides performance close to that of genius aided ranging refinement (columns B and C). Ranging refinement provides a performance improvement greater than cooperation, and this might depend on the particular cooperative setting considered. The impact of combined cooperation and ranging refinement with multilevel NLOS identification is shown in columns D, $\mathrm{E}$, and $\mathrm{F}$.

Fig. 3 shows the LEO for various target localization RMSE $e_{\text {th }}$ for cases D, E, and F. As example, with WED identification ranging refinement LEO is $10 \%$ with $e_{\text {th }}$ about $40 \mathrm{~cm}$, which means that in $90 \%$ of cases the RMSE is not greater than 40 $\mathrm{cm}$, slightly above the value obtained with WED genius aided. With no ranging refinement, the LEO of $10 \%$ is obtained with a localization RMSE of $60 \mathrm{~cm}$.

\section{CONClusion}

In this paper, we provide experimental results on cooperative and non-cooperative localization in a real indoor environment. It is shown that ranging refinement based on NLOS identification is beneficial for localization performance, and a multilevel NLOS characterization technique is proposed. It can utilize various waveforms features and combination of them to recognize the number of walls between each pair of nodes which affects the ranging estimation. Localization RMSE and error outage are given for UWB devices, based on which a system designer can understand how to exploit cooperation and ranging refinement.

\section{ACKNOWLEDGMENTS}

This research was supported in part by the FP7 European Project Select (Grant 257544) and Ministero dello Sviluppo Economico under the Project WEBS. The authors wish to thank Proff. D. Dardari and M. Z. Win for helpful discussions.

\section{REFERENCES}

[1] R. A. Scholtz and M. Z. Win, "Impulse radio," in Wireless Communications: TDMA versus CDMA, S. G. Glisic and P. A. Leppänen, Eds. Norwell, MA 02061: Kluwer Academic Publishers, 1997, ch. 2.7, pp. 245-263.

[2] S. Gezici, Z. Tian, G. B. Giannakis, H. Kobayashi, A. F. Molisch, H. V. Poor, and Z. Sahinoglu, "Localization via ultra-wideband radios: a look at positioning aspects for future sensor networks," IEEE Signal Process. Mag., vol. 22, no. 4, p. 7084, Jul. 2005.

[3] A. F. Molisch, "Ultrawideband propagation channels-theory, measurement, and modeling," IEEE Trans. Veh. Technol., vol. 54, no. 5, p. 15281545, Sep. 2005

[4] Y. Shen and M. Z. Win, "On the accuracy of localization systems using wideband antenna arrays," IEEE Trans. Commun., vol. 58, no. 1, pp. 270-280, Jan. 2010

[5] — , "Fundamental limits of wideband localization - Part I: A general framework," IEEE Trans. Inf. Theory, vol. 56, no. 10, pp. 4956-4980, Oct. 2010. [Online]. Available: http://arxiv.org/abs/1006.0888v1

[6] D. Dardari, A. Conti, U. J. Ferner, A. Giorgetti, and M. Z. Win, "Ranging with ultrawide bandwidth signals in multipath environments," Proc. IEEE, vol. 97, no. 2, pp. 404-426, Feb. 2009, special issue on UltraWide Bandwidth (UWB) Technology \& Emerging Applications.

[7] J.-Y. Lee and R. A. Scholtz, "Ranging in a dense multipath environment using an UWB radio link," IEEE J. Sel. Areas Commun., vol. 20, no. 9 pp. 1677-1683, Dec. 2002.

[8] D. Dardari, A. Conti, J. Lien, and M. Z. Win, "The effect of cooperation on localization systems using UWB experimental data," EURASIP J. Appl. Signal Process., vol. 2008, pp. Article ID 513873, 1-11, 2008, special issue on Cooperative Localization in Wireless Ad Hoc and Sensor Networks.

[9] I. Güvenç, C.-C. Chong, F. Watanabe, and H. Inamura, "NLOS identification and weighted least-squares localization for UWB systems using multipath channel statistics," EURASIP J. Adv. in Signal Process., vol. 2008, pp. 1-14, 2008.

[10] J. Schroeder, S. Galler, K. Kyamakya, and K. Jobmann, "NLOS detection algorithms for ultra-wideband localization," Mar. 2007, pp. 159166.

[11] S. Maranò, W. M. Gifford, H. Wymeersch, and M. Z. Win, "NLOS identification and mitigation for localization based on UWB experimental data," IEEE J. Sel. Areas Commun., vol. 28, no. 7, pp. 1026-1035, Sep. 2010

[12] S. Gezici, H. Kobayashi, and H. V. Poor, "Non-parametric non-lineof-sight identification," in Proc. IEEE Semiannual Veh. Technol. Conf. (VTC), vol. 4, Orlando, FL, Oct. 2003, pp. 2544-2548.

[13] A. Maali, H. Mimoun, G. Baudoin, and A. Ouldali, "A new low complexity NLOS identification approach based on UWB energy detection," San Diego, CA, Jan. 2009, pp. 675-78.

[14] N. Patwari, J. N. Ash, S. Kyperountas, A. O. Hero, III, R. L. Moses, and N. S. Correal, "Locating the nodes: cooperative localization in wireless sensor networks," IEEE Signal Process. Mag., vol. 22, no. 4, pp. 54-69, Jul. 2005.

[15] M. Z. Win and A. Conti, "Network localization and navigation via cooperation," IEEE Commun. Mag., no. 5, May 2011.

[16] B. Alavi and K. Pahlavan, "Modeling of the TOA-based distance measurement error using UWB indoor radio measurements," IEEE Commun. Lett., vol. 10, no. 4, pp. 275-277, Apr. 2006.

[17] H. Wymeersch, J. Lien, and M. Z. Win, "Cooperative localization in wireless networks," Proc. IEEE, vol. 97, no. 2, pp. 427-450, Feb. 2009, special issue on Ultra-Wide Bandwidth (UWB) Technology \& Emerging Applications.

[18] I. Guvenc, C.-C. Chong, and F. Watanabe, "NLOS identification and mitigation for UWB localization systems," in Proc. IEEE Wireless Commun. and Networking Conf. (WCNC), Kowloon, China, Mar. 2007, pp. 1571-1576.

[19] A. Conti, M. Z. Win, M. Chiani, and J. H. Winters, "Bit error outage for diversity reception in shadowing environment," IEEE Commun. Lett., vol. 7 , no. 1 , pp. $15-17$, Jan. 2003. 